Endometriumkarzinom

\title{
Vorteil durch Sentinel-Biopsie?
}

Metastasen des Endometriumkarzinoms lassen sich mithilfe der Wächterlymphknotenbiopsie offenbar mit hoher Genauigkeit vorhersagen. Beim Staging des Karzinoms könnte sie den betroffenen Frauen eine Lymphonodektomie ersparen, so das Fazit der prospektiven Studie FIRES. In der Studie mit 385 Patientinnen im medianen Alter von 63 Jahren wurde jeweils in die Ektozervix bei 3 und 9 Uhr bis zu einer Tiefe von $1 \mathrm{~cm}$ der Farbstoff Indocyanin injiziert. Nach der robotergestützten Kartierung erfolgte bei 340 Frauen eine komplette pelvine Lymphonodektomie. Nur bei etwas mehr als der Hälfte (58\%) wurden paraaortale Lymphknoten entfernt.

41 Patientinnen (12\%) hatten ein metastasiertes Endometriumkarzinom, nachgewiesen anhand der Wächterlymphknoten oder der durch Lymphonodektomie erhaltenen Proben. Bei 84\% der Patientinnen entfernten die Chirurgen mindestens zehn Lymphknoten. Jeweils

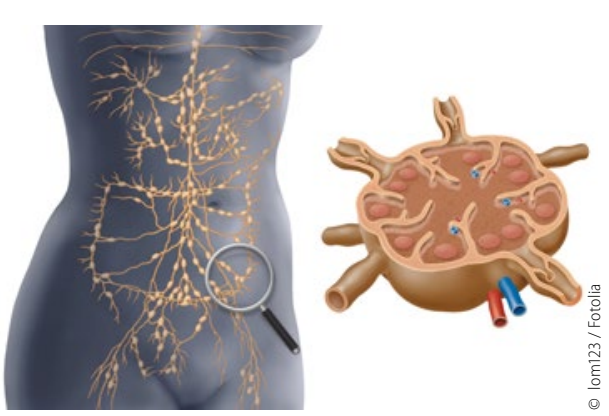

Die Sentinelbiopsie könnte auch beim Endometriumkarzinom sinnvoll sein. mindestens einen positiven Wächterlymphknoten identifizierten sie bei $86 \%$. 888 Wächterlymphknoten wurden mithilfe der Färbemethode lokalisiert. 36 der 293 Patientinnen mit mindestens einem positiven Wächterlymphknoten hatten Metastasen in weiteren Lymphknoten, 35 (97\%) von ihnen wurden anhand der Wächterlymphknoten korrekt diagnostiziert.

Aufgrund dieser Befunde errechneten die Ärzte eine Sensitivität für die Sentinel-Lymphknoten-Biopsie beim Nachweis einer Metastasierung der Lymphknoten von 97,2\%. Bei 257 der 258 Frauen mit negativen Wächterlymphknoten waren alle anderen Lymphknotenbiopsien ebenfalls negativ. Der negative prädiktive Wert liegt demnach bei 99,6\%.

Peter Leiner
Rossi EC et al. A comparison of sentinel lymph
node biopsy to lymphadenectomy for endometrial cancer staging (FIRES trial): a multicentre, prospec- tive, cohort study. Lancet Oncol. 2017;18(3):384-92.

Fortgeschrittenes Mammakarzinom

\section{Hormonblockade und was dann?}

Die Addidition des CDK-4/6-Inhibitors Palbociclib zur endokrinen Erstlinientherapie des fortgeschrittenen Östrogenrezeptor(ER)-positiven, HER2-negativen Mammakarzinoms verlängert signifikant das progressionsfreie Überleben. Die Patienten leiden jedoch häufiger unter myelotoxischen Nebenwirkungen. Das zeigte die Phase-III-Studie PALOMA-2. 666 postmenopausale Frauen mit fortgeschrittenem ER-positivem, HER2negativem Brustkrebs, die bislang keine andere Therapie für dieses Erkrankungsstadium erhalten hatten, wurden entweder mit Palbociclib plus Letrozol oder mit Palbociclib plus Placebo behandelt.

Das mediane progressionsfreie Überleben lag in der Palbociclib/Letrozol-Gruppe mit 24,8 Monaten signifikant über dem in der Kontrollgruppe mit 14,5 Monaten. Die Hazard Ratio (HR) für Krankheitsprogression oder Tod betrug 0,58. Der Vorteil der Palbociclib-Addition zeigte sich in allen Subgruppen und war damit unabhängig von der Metastasenlokalisation, dem Alter, dem Performancestatus, der Vortherapie oder der Histopathologie.
Die Kombination Palbociclib/Letrozol führte signifikant häufiger zu unerwünschten Nebenwirkungen myelotoxischer Natur: Neutropenie (66,4 vs. $1,4 \%)$, Leukopenie $(24,8$ vs. $0 \%$ ) und Anämie (5,4 vs. $1,8 \%)$. Febrile Neutropenien traten jedoch nur selten auf (1,8 vs. $0 \%)$. Nicht bestätigt wurde die zuvor gesehene
Tendenz zu einem häufigeren Auftreten von Lungenembolien (0,9 vs. 1,4\%). Auch schwere Grad-3-Infektionen traten in beiden Gruppen vergleichbar häufig auf. Ein Abbruch der Therapie aufgrund behandlungsbedingter Nebenwirkungen erfolgte öfter in der Palbociclib/LetrozolGruppe (9,7 vs. 5,9\%), dieser Unterschied war jedoch nicht signifikant. AnnaAtak

Finn RS et al. Palbociclib and Letrozole in Advanced Breast Cancer. N Engl J Med. 2016;375(20): 1925-36.

\section{kurz notiert}

\section{Wie Brokkoli gegen Prostatakrebs wirkt}

Sulforaphan, ein Inhaltsstoff von Brokkoli, schützt möglicherweise über den Einfluss auf lange, nicht kodierende RNAs (IncRNA) vor Prostatakrebs. Das haben Wissenschaftler der Oregon State University in den USA herausgefunden. LncRNAs haben häufig Einfluss darauf, welche Gene eingeschaltet bzw. exprimiert werden. Offensichtlich spielen sie auch eine entscheidende Rolle dabei, ob sich Zellen zu malignen Zellen entwickeln und streuen. Die Forscher hatten das Genom von humanen Prostatakrebszellen analysiert. Sulforaphan veränderte die Expression von etwa 100 IncRNAs in jedem Zelltyp und normalisierte die Expression einiger IncRNAs, deren Expression in Krebszellen verändert war. LINC01116 ist eine IncRNA, die in humanen Prostatakarzinom-Zelllinien hochreguliert ist, aber durch die Gabe von Sulforaphan reduziert werden kann. Wenn LINC01116 mithilfe des CRISPR/CAS9-Systems beeinträchtigt wurde, nahm die Fähigkeit von Prostatakrebszellen, Kolonien zu bilden, um das Vierfache ab, was die onkogene Funktion von LINC01116 in Prostatakrebszellen bestätigt. Damit zeigen diese Daten, dass IncRNAs einen interessanten neuen Weg für die Forschung zur Krebsprävention oder der Verlangsamung der Progression von Tumorzellen eröffnen und dass Substanzen aus der Nahrung deren Expression beeinflussen können [Beaver LM et al. J Nutr Biochem. 2017;42:72-83]. Judith Neumaier 